http://jmscr.igmpublication.org/home/ ISSN (e)-2347-176x ISSN (p) 2455-0450

crossref DOI: https://dx.doi.org/10.18535/jmscr/v8i2.89

\title{
To Study the Dry Eye Prevalence Following Phacoemulsification Surgery at Tertiary Care Hospital Rajasthan
}

\begin{abstract}
Authors
Dr Sukriti Upadhyay ${ }^{1}$, Dr Udit Raj Sharma ${ }^{2}$

${ }^{1}$ Junior Resident, III year, Upgraded Department of Ophthalmology, SMS Medical College \& Hospital, Jaipur, India

${ }^{2}$ Senior Resident, Upgraded Department of Ophthalmology, SMS Medical College \& Hospital, Jaipur, India Corresponding Author

Dr Udit Raj Sharma

Abstract

Background: The purpose was to study dry eye following phacoemulsification surgery and analyze it's relation to the associated intra-operative risk factors.

Methods: This prospective observational study was performed after selecting 100 consecutive patients who attended the outpatient Department of Ophthalmology, SMS Medical College and Hospital, Jaipur. Prior informed consent was obtained from each subject, inclusion criteria being subjects over 45 years of age with senile cataract, without preexisting dry eye and other ocular disorders. Subjects with disorders of eyelids, past ocular surgeries, pre-existing ocular or systemic diseases, history of taking ocular or systemic medications and smoking were excluded from the study.

Results: The present study was done on 100 patients attending the outpatient Department of Ophthalmology, SMS Medical College and Hospital, Jaipur with senile cataract and each patient was scheduled for cataract surgery using phacoemulsification technique. All the tests performed preoperatively and postoperatively showed incidence of dry eye to be 10.00\%. Dry eye symptoms peaked at day 7 in our study.

Conclusion: We concluded that cataract surgery can cause dry eye. However, before surgery, preoperative assessment of all patients should be done properly. Patients must be informed about the possible occurrence of dry eye symptoms postoperatively and should be managed accordingly.

Keywords: Dry Eye, Schirmer Test, Tear Film Break Up Time, OSDI.
\end{abstract}

\section{Introduction}

A disorder of the tear film due to tear deficiency or excessive evaporation, which causes damage to the interpalpebral ocular surface and is associated with symptoms of ocular discomfort is Dry eye is an under-diagnosed ocular disorder in middle aged and older adults. The prevalence of dry eye varies from $10.8 \%$ to $57.1 \%$. It has been documented that symptoms of dry eye. ${ }^{1}$
Prevalence varied from $8.4 \%$ in less than 60 yrs to $19.0 \%$ in those older than $80 \mathrm{yrs}^{2}$. Currently, available diagnostic tests do not correlate reliably with symptom severity of dry eye. Prevalence of 1 or more of the 6 dry eye symptoms adjusted for age was $27.5 \% .^{3}$

Tear secretion may also be decreased by any condition which decreases corneal sensation, including common entities such as Herpes Zoster, 
long term contact lens wear and ocular surgeries that involve corneal incisions like cataract extraction or those which ablate corneal nerves like laser in situ keratomileusis (LASIK). ${ }^{4}$

Denervation of the corneal nerves by incision, production of free radicals due to ultrasound energy, microscope light exposure time during surgery and pre- and post-operative medications have been implicated as the possible causes of dry eye disease following phacoemulsification surgery.

\section{Materials and Methods}

This prospective observational study was performed after selecting 100 consecutive patients who attended the outpatient Department of Ophthalmology, SMS Medical College and Hospital, Jaipur.

Prior informed consent was obtained from each subject, inclusion criteria being subjects over 45 years of age with senile cataract, without preexisting dry eye and other ocular disorders. Subjects with disorders of eyelids, past ocular surgeries, pre-existing ocular or systemic diseases, history of taking ocular or systemic medications and smoking were excluded from the study.

General patient information and detailed history of systemic and ocular diseases was recorded, and thorough evaluation was carried out in all the patients. 2 days before the cataract surgery, ST-I, TBUT, and TMH were measured. First, ST-I was evaluated without corneal anesthesia by using a standardized tear strip (Bio division Ltd., UK).

In a quiet room, with dimmed light, the patient was made to sit comfortably and the strip was inserted into the lower temporal lid margin, after folding it at the notch and asking the patient to look up and in. After $5 \mathrm{~min}$, the strip was removed, and the length of the moistened area was recorded. Next, the stability of the tear film over the conjunctiva and cornea, TBUT, was assessed using a slit-lamp with a cobalt blue filter and sodium fluorescein. The interval between ST-I and TBUT was at least $10 \mathrm{~min}$.
A fluoresce in strip was applied in the lower palpebral conjunctiva, and the patient was asked to blink 5 times, after which he/she was asked to refrain from blinking. The appearance of black spots or lines indicated the onset of dry spots and the interval between the last blink and the first randomly distributed dry spot was taken as the TBUT. The average of three measurements was recorded and a value $<10$ seconds was taken as abnormal. After 5 minutes, with the patient sitting on the same slit lamp, TMH was evaluated by adjusting the vertical length of slit beam on the tear meniscus at the center of the lower lid and the readings were noted from the slit lamp scale.

Lissamine green staining of the cornea and conjunctiva was done on the next day. For this, LG filter paper was applied in the lower palpebral conjunctiva and the patient was asked to blink several times. The grading was done as per 0-5 scale Oxford grading scheme of LG staining of cornea and conjunctiva, using a slit lamp, set at $\times 16$ magnification with $\times 10$ oculars with HaagStreit slit lamp.

\section{Results}

In our study, the average age was $61.24 \pm 5.13$ years (range: 43-77). There were more females $(62.00 \%)$ as compared to males $(38.00 \%)$.

On preoperative examination, the mean of Schirmer I test was $20.31 \pm 6.21$, on day $1-19.32$ \pm 5.11 , On day $7-18.56 \pm 5.23$, On Day $30-$ $19.58 \pm 5.89$, On Day $90-20.16 \pm 6.08$. 


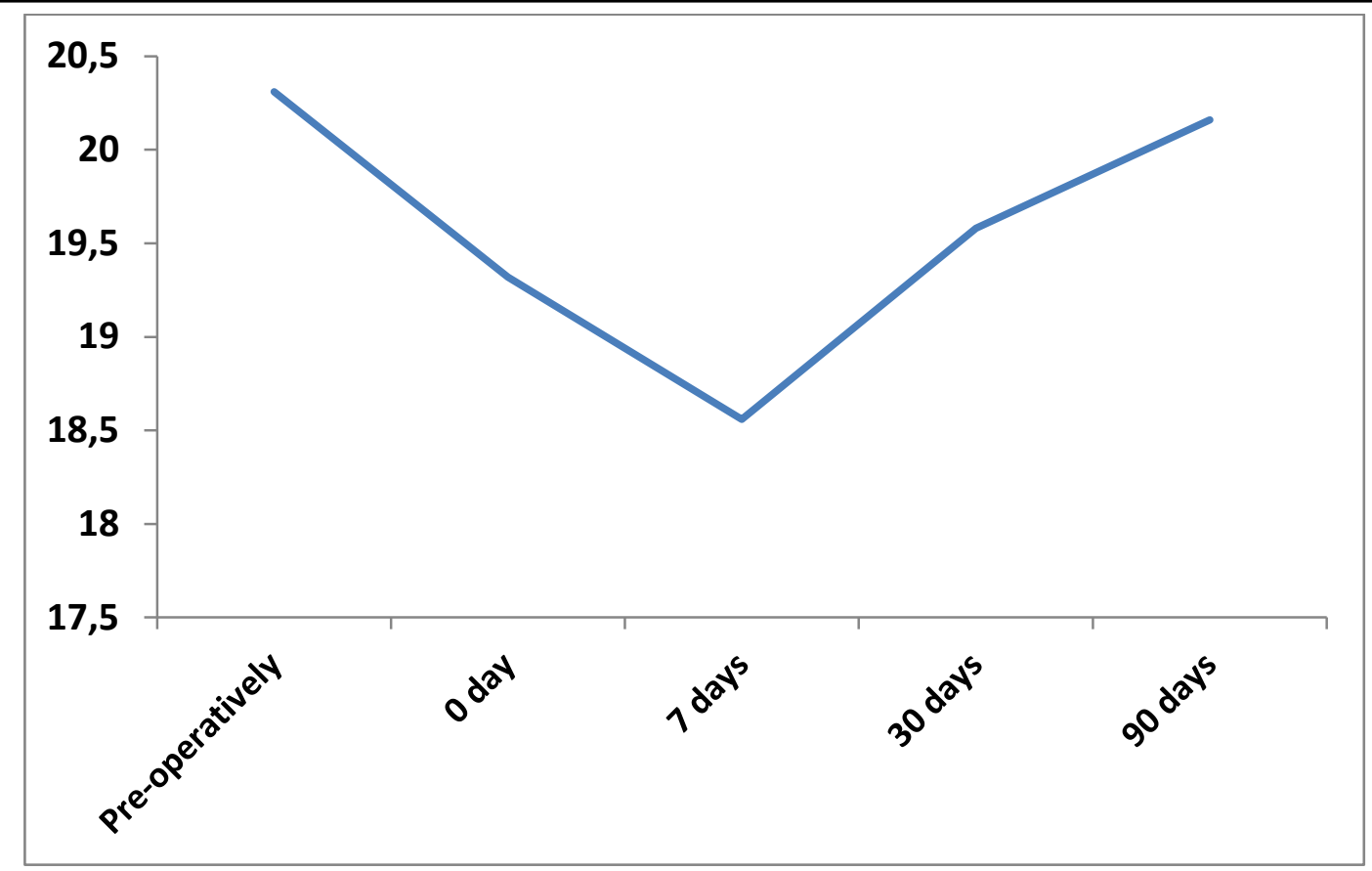

Figure 1: Schirmer 1 Test Pattern: pre operative and post operative

On preoperative examination, the mean of Schirmer II scores was $18.56 \pm 1.05$. On day 0 , the mean was $17.02 \pm 1.12$. On day 7 , the mean was $16.04 \pm 1.03$. On day 30 , the mean was 16.32 \pm 0.98 . On day 90 , the mean of Schirmer II score was $16.96 \pm 0.67$. According to pattern of Schirmer 2 scores, dry eye patients peaked at day 7 and scores gradually improved over follow up.

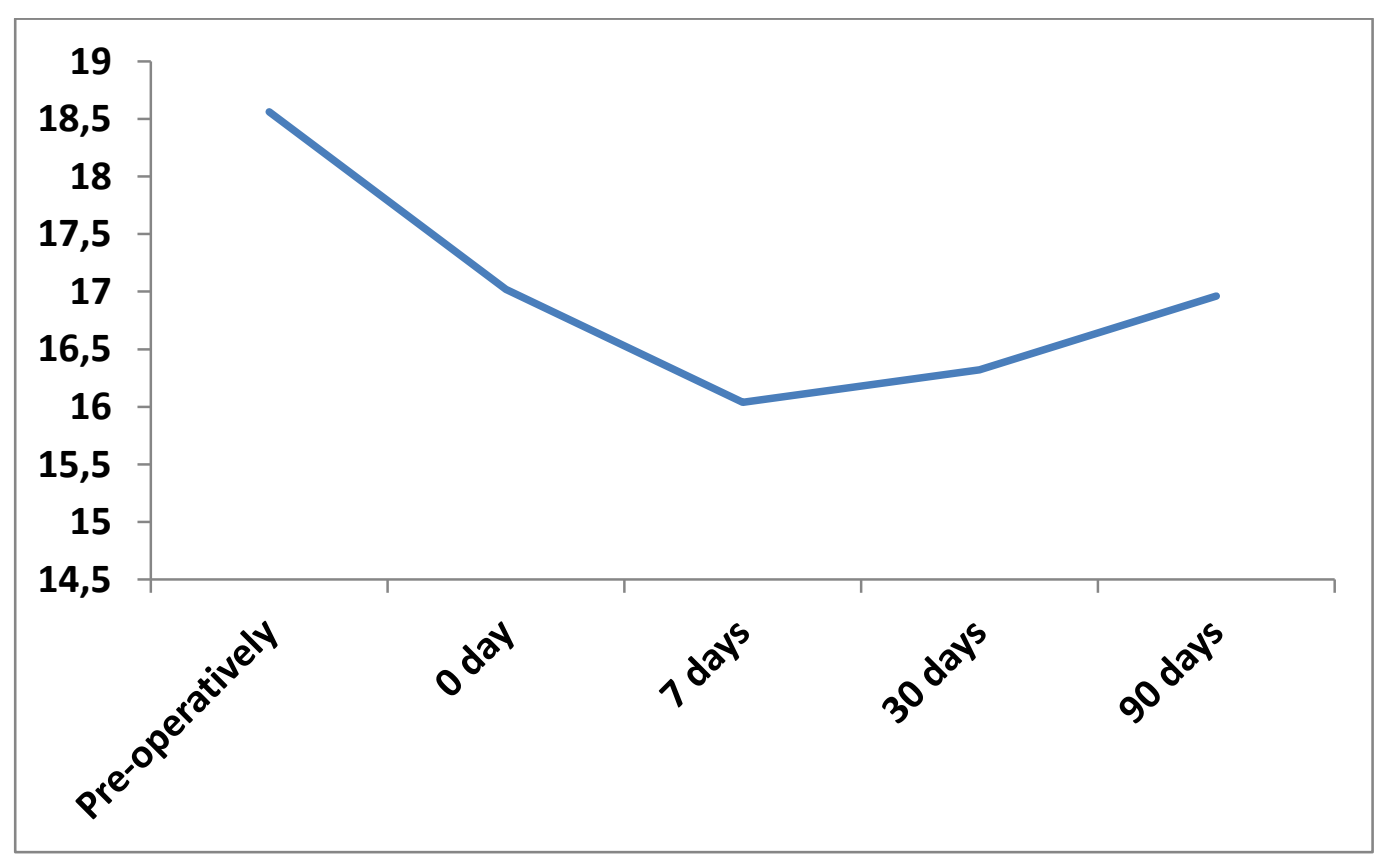

Figure 2: Schirmer 2 Test Pattern: pre operative and post operative

On preoperative examination, the mean of Tear Film Break up Time scores was $13.26 \pm 0.47$. On day 1 , the mean was $12.10 \pm 0.52$. On day 7 , the mean was $10.23 \pm 0.64$. On day 30 , the mean was $11.46 \pm 0.71$. On day 90 , the mean of tear film break up scores was $11.89 \pm 0.61$. According to the pattern of tear film break up scores, dry eye patients peaked at day 7 and the scores gradually improved over follow up days. 


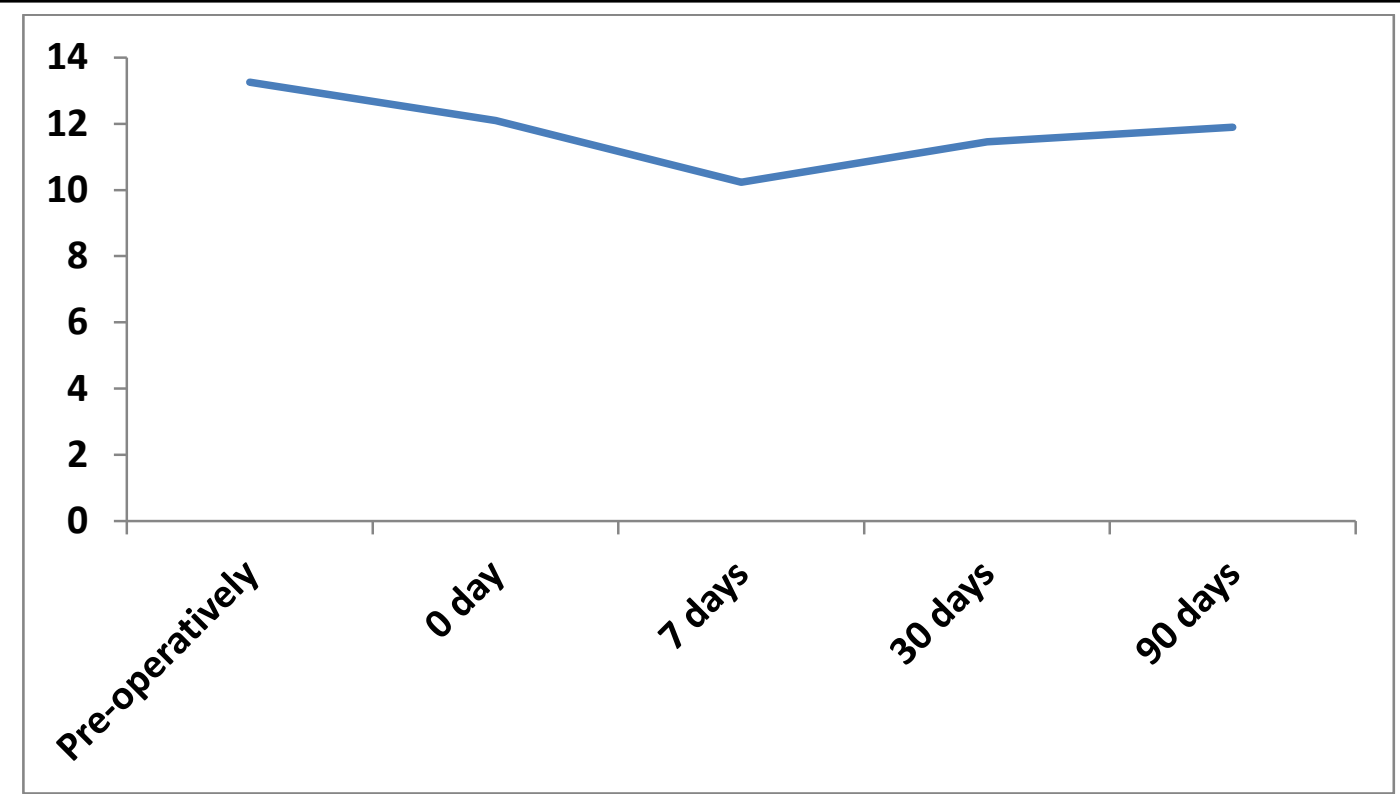

Figure 3: Tear Film Break Up Time: pre operative and post operative

On preoperative examination, the mean of Ocular Surface Disease Index scores was $13.12 \pm 0.52$. On day 1 , the mean was $13.09 \pm 0.59$. On day 7 , the mean was $26.52 \pm 1.89$. On day 30 , the mean was $23.13 \pm 1.52$. On day 90 , the mean of OSDI score was $19.22 \pm 1.06$. According to pattern of Ocular Surface Disease Index scores, dry eye patients peaked at day 7 and scores remained nearly the same on follow up.

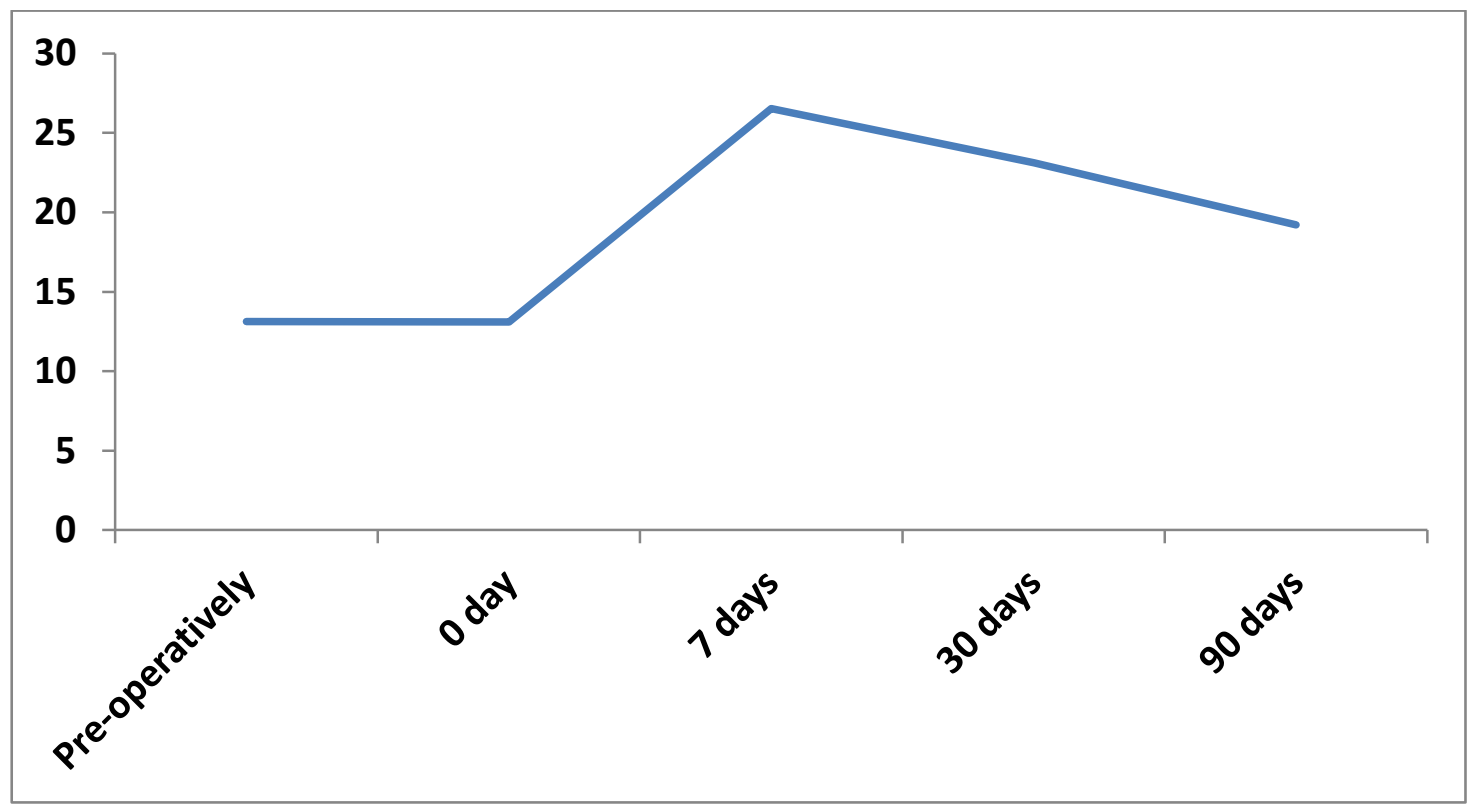

Figure 4: Ocular Surface Disease Index: pre operative and post operative

On preoperative examination, the mean value of Rose Bengal staining scores was $0.36 \pm 0.05$. On day 1 , the mean was $0.71 \pm 0.155$. On day 7 , the mean was $0.95 \pm 0.218$. On day 30 , the mean was $0.81 \pm 0.183$. On day 90 , the mean value of Rose Bengal staining scores was $0.67 \pm 0.13$. According to pattern of Rose Bengal staining, dry eye patients on day 7 had confluent staining with more number of patients having higher scores and on follow up days, the staining scores of patients decreased. 


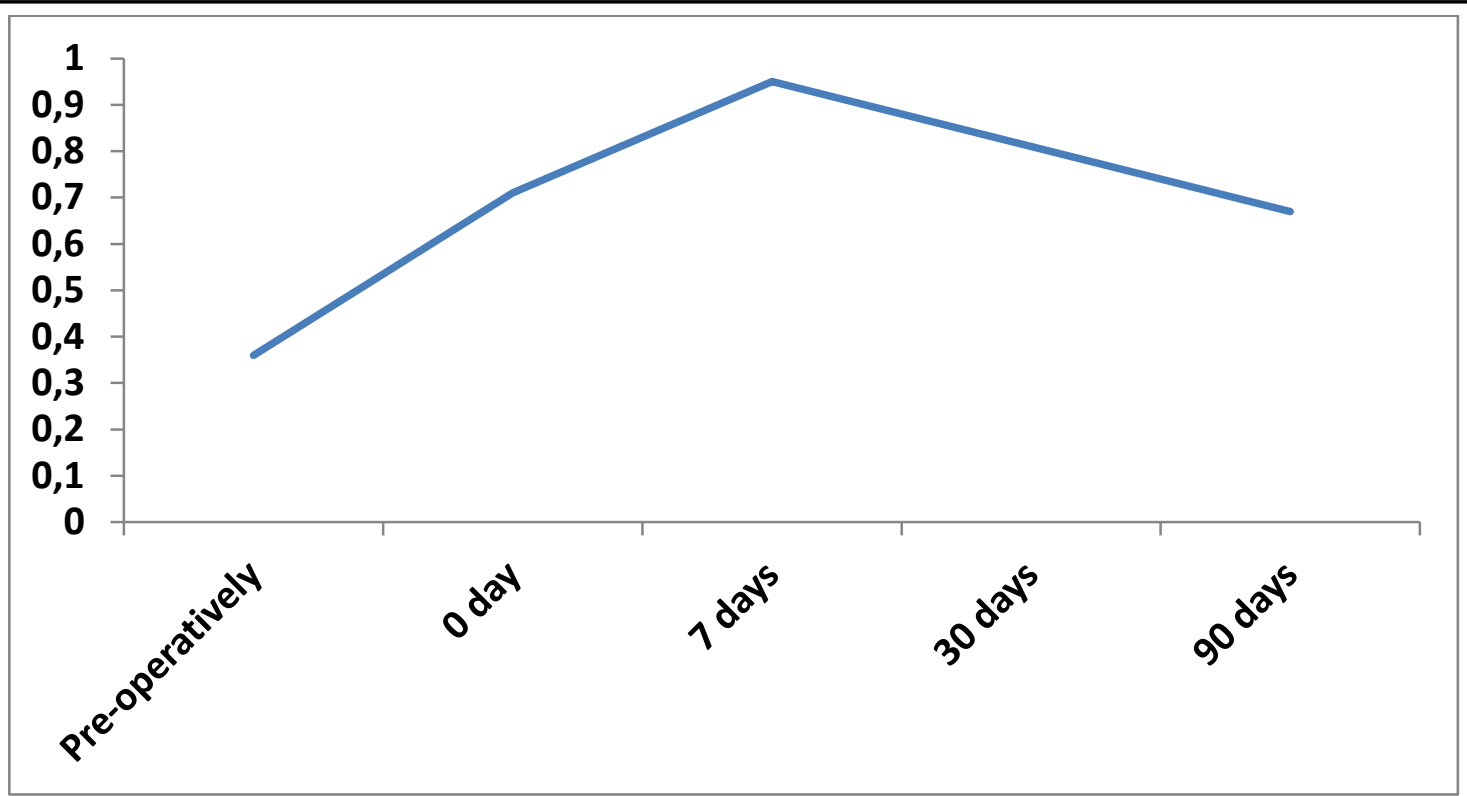

Figure 5: Rose Bengal staining scores: pre operative and post operative

Table 1 Association between age and dry eye

\begin{tabular}{|l|c|c|c|}
\hline \multirow{2}{*}{$\begin{array}{l}\text { Age } \\
\text { group }\end{array}$} & \multicolumn{2}{|c|}{ Dry eye } & \multirow{2}{*}{ Total } \\
\cline { 2 - 3 } & Present & Absent & \\
\hline$\leq 50$ & 2 & 10 & 12 \\
\hline $51-60$ & 4 & 27 & 31 \\
\hline $61-70$ & 3 & 41 & 44 \\
\hline$>70$ & 1 & 12 & 13 \\
\hline Total & 10 & 90 & 100 \\
\hline
\end{tabular}

In the age group of up to 50 years, out of 12 , two patients developed dry eye and 10 patients had no dry eye. In the age group of 51 to 60 years, out of 31,4 patients developed dry eye and 27 patients had no dry eye. In the age group of 61 to 70 years, out of 44 , three patients developed dry eye and 41 patients had no dry eye. In the age group of $>70$ years, out of 13, 1patient developed dry eye and 12 patients had no dry eye. Chi square value was 2.124 and $p$ value was 0.516 .

Table 2 Association between Sex and dry eye

\begin{tabular}{|l|c|c|c|}
\hline \multirow{2}{*}{ Sex } & \multicolumn{2}{|c|}{ Dry eye } & \multirow{2}{*}{ Total } \\
\cline { 2 - 3 } & Present & Absent & \\
\hline Male & 3 & 35 & 38 \\
\hline Female & 7 & 55 & 62 \\
\hline Total & 10 & 90 & 100 \\
\hline
\end{tabular}

Out of thirty eight female patients, three patients developed dry eye and thirty three patients had no dry eye. Out of sixty two male patients, seven patients developed dry eye and fifty five patients had no dry eye. Chi square value was 0.312 and $p$ value was 0.612 . Hence, no association was found between sex of the patient and development of dry eye. Development of dry eye was equally common in both males and females.

\section{Discussion}

Dry eye can develop often after various types of ophthalmic surgeries such as photorefractive keratectomy and laser-assisted in situ keratomileusis (LASIK). After LASIK, dry eye can persist for up to 6 months or more with an incidence of $20 \% .^{5}$

Various factors might affect the ocular surface environment after cataract surgery. Most important is corneal desensitization. ${ }^{6}$

Superficial punctate keratitis, recurrent filamentary keratitis, secondary infections including conjunctivitis, infective keratitis, persistent or recurrent epithelial defects, stromal keratolysis and corneal ulceration have been reported in dry eye patients after cataract surgery notably conventional extracapsular cataract extraction (ECCE) by Ram et al in 2002. ${ }^{7}$

The present study was conducted on 100 patients attending the outpatient Department of Ophthalmology, SMS Medical College and Hospital, Jaipur with senile cataract who all underwent cataract surgery using phacoemulsification technique. All the tests 
performed preoperatively and postoperatively showed incidence of dry eye to be $10.00 \%$.

Dry eye symptoms peaked at day 7 in our study. Another explanation for the dry eye pattern observed in the current study was the recovery process of the corneal nerves. The cornea is one of the most highly innervated organs, with about 44 corneal nerve bundles entering the cornea around the limbus centripetally ${ }^{8}$ and larger nerve fibers that run from the 9 o'clock to the 3 o'clock position and bifurcate to achieve a homogenous distribution over the entire cornea. ${ }^{9}$ Therefore, it is vulnerable to damage within that region. Temporal corneal incisions created during phacoemulsification can reduce the corneal sensitivity in the surgical area and other areas far from the incision site. ${ }^{10}$ Damage to the corneal nerves may increase when more phacoemulsification time is needed to emulsify a dense cataract.

In addition to transection of the corneal nerves and damage to the corneal epithelial cells, exposure to microscopic light, vigorous intra operative irrigation of the tear film, elevation of inflammatory factors in the tear film due to ocular surface irritation, use of topical anesthesia intraoperatively and topical eye drops administered postoperatively and its preservatives can cause dry eye after phacoemulsification. ${ }^{11,12}$

\section{Conclusion}

We concluded that cataract surgery can cause dry eye. However, before surgery, preoperative assessment of all patients should be done properly. Patients must be informed about the possible occurrence of dry eye symptoms postoperatively and should be managed accordingly.

\section{References}

1. Vankadara Naga Suresh, Radhika. THE PREVALENCE OF DRY EYE IN TERTIARY HEALTH CARE CENTER
OF ANDHRA PRADESH. Int J Intg Med Sci 2018;5(3):592-596.

2. Moss SE, Klein R, Klein BEK. Prevalence of and risk factors for dry eye syndrome. Arch Ophthalmol. 2000;118:1264-68.

3. Smith, Janine, Nichols, Kelly k, Baldwin, Edward k. Current patterns in the use of diagnostic tests in dry eye evaluation. Cornea 2008;27(6):656-62.

4. Roberts CW, Elie ER. Dry eye symptoms following cataract surgery. Insight 2007;32:14-21.

5. Shoja MR, Besharati MR. Dry eye after LASIK for myopia: Incidence and risk factors. Eur J Ophthalmol 2007; 17:1-6.

6. Ministry of Health and Family Welfare, Govt. Of India. National Programme for Control of Blindness, Annual Report, MOHFW. Govt of India 2010-11; 109-11.

7. Ram J, Gupta A, Brar GS, Kaushik S, Gupta A. Outcomes of phacoemulsification in patients with dry eye. J Cataract Refract Surg 2002; 28:1386-89.

8. Muller LJ, Vrensen GF, Pels L, Cardozo BN, Willekens B. Architecture of human corneal nerves. Invest Ophthalmol Vis Sci 1997; 38:985-94.

9. Sitompul R, Sancoyo GS, Hutauruk JA, Gondhowiardjo TD. Sensitivity change in cornea and tear layer due to incision difference on cataract surgery with either manual small-incision cataract surgery or phacoemulsification. Cornea 2008; (Suppl 1): S13-8.

10. Belmonte C, Acosta MC, Gallar J. Neural basis of sensation in intact and injured corneas. Exp Eye Res 2004; 78:513-25.

11. Roberts CW, Elie ER. Dry eye symptoms following cataract surgery. Insight 2007; 32:14-21.

12. Liu ZG, Li W. Dry eye relevant to ocular surgery. Zhonghua Yan KeZaZhi 2009; 45:483-5. 